\title{
Narguilé: consumo e conhecimento entre acadêmicos de Odontologia
}

\author{
Hookah: consumption and knowledge among Dentistry students \\ Hookah: consumo y conocimiento entre estudiantes de Odontología
}

\section{Resumo}

O consumo de tabaco através narguilé pela população jovem aumentou nos últimos anos no Brasil. Neste estudo, buscou-se identificar a prevalência do consumo e o perfil dos usuários de narguilé entre os acadêmicos de Odontologia. Trata-se de uma pesquisa com abordagem quantitativa, realizada por meio de questionários autoaplicáveis abordando o hábito de fumar e conhecimento sobre narguilé. Concordaram em participar do estudo 160 acadêmicos matriculados no curso de Odontologia da Universidade Estadual do Paraná (UNIOESTE), no ano de 2016. De acordo com os dados coletados, $69 \%(\mathrm{n}=111)$ dos acadêmicos entrevistados já haviam experimentado o narguilé, sendo que 59\% ( $n=66)$ desses iniciaram o uso na faixa etária entre 17 e 19 anos e $41 \%(n=45)$ entre 20 e 24 anos. Para $45 \%(\mathrm{n}=50)$ dos participantes deste estudo, a curiosidade foi responsável pelo contato com o narguilé, e para 23\% ( $\mathrm{n}=25)$ estar na universidade propiciou o uso dessa substância. A influência dos amigos motivou 77\% a experimentar o narguilé e a associação desse tipo de tabaco com drogas ilícitas foi relatado por 8\% (9/111) dos usuários. Em relação aos conhecimentos dos acadêmicos sobre o assunto, $52 \%(\mathrm{n}=83)$ acreditam que a fumaça do cigarro possui mais substâncias tóxicas quando comparada com o narguilé. Assim, os achados enfatizam a necessidade de investigar e desenvolver melhores formas de conscientizar os acadêmicos acerca dos componentes encontrados ou produzidos por esse tipo de fumo e das doenças associadas a essa prática.

Palavras-chave: Fumar cachimbo de água; Cachimbo de água; Tabagismo.

\begin{abstract}
Tobacco consumption through hookah by the young population has increased in recent years in Brazil. In this study, we sought to identify the prevalence of consumption and the profile of hookah users among dentistry students. This is a research with a quantitative approach, carried out through self-administered questionnaires addressing the smoking habit and knowledge about hookah. 160 academics enrolled in the Dentistry course at the State University of Paraná (UNIOESTE) in 2016 agreed to participate in the study. According to the data collected, 69\% $(n=111)$ of the academics interviewed had already tried the hookah. 59\% $(n=66)$ of these started using between 17 and 19 years old and $41 \%(n=45)$ between 20 and 24 years old. For $45 \%(n=50)$ of the participants in this study, curiosity was responsible for the contact with the hookah, and for $23 \%(\mathrm{n}=25)$ being at the university provided the use of this
\end{abstract}


substance. The influence of friends motivated $77 \%$ to try the hookah, and the association of this type of tobacco with illicit drugs was reported by $8 \%$ (9/111) of the participants. Regarding the knowledge of academics on the subject, $52 \%(\mathrm{n}=83)$ said they believed that, unfortunately, cigarette smoke has more toxic substances when compared to hookah. Thus, the findings emphasize the need to investigate and develop better ways to make students aware of the components found or produced by this type of tobacco and the diseases associated with this practice.

Keywords: Water pipe smoking; Smoking water pipes; Tobacco use disorder.

\section{Resumen}

El consumo de tabaco a través de narguile por parte de la población joven se ha incrementado en los últimos años en Brasil. En este estudio, buscamos identificar la prevalencia del consumo y el perfil de usuarios de narguile entre los estudiantes de odontología. Se trata de una investigación con enfoque cuantitativo, realizada a través de cuestionarios autoadministrados que abordan el hábito de fumar y los conocimientos sobre narguile. 160 académicos matriculados en el curso de Odontología de la Universidad Estatal de Paraná (UNIOESTE) en 2016 aceptaron participar en el estudio.De acuerdo con los datos recolectados, el $69 \%(\mathrm{n}=111)$ de los académicos entrevistados ya había probado la pipa de agua. 59\% $(n=66)$ de estos empezaron a consumir entre los 17 y 19 años y el $41 \%(n=45)$ entre los 20 y 24 años. Para el $45 \%(n=50)$ de los participantes en este estudio, la curiosidad fue responsable del contacto con la pipa de agua, y para el $23 \%(\mathrm{n}=25)$ estar en la universidad proporcionó el uso de esta sustancia. La influencia de los amigos motivó al $77 \%$ a probar la pipa de agua, y la asociación de este tipo de tabaco con drogas ilícitas fue reportada por el 8\% (11/9) de los participantes. En cuanto al conocimiento de los académicos sobre el tema, el 52\% (n= 83) dijo creer que, lamentablemente, el humo del cigarrillo tiene más sustancias tóxicas en comparación con el narguile. Así, los hallazgos enfatizan la necesidad de investigar y desarrollar mejores formas de concienciar a los estudiantes sobre los componentes encontrados o producidos por este tipo de tabaco y las enfermedades asociadas a esta práctica.

Palabras clave: Fumar en pipa de agua; Pipas de agua; Tabaquismo.

\section{Introdução}

O narguilé, também conhecido como cachimbo d'água, arguilé, sisha ou hookah é um instrumento de origem oriental desenvolvido por um médico durante o reinado do imperador Akbar (1556-1605), acreditando-se que as substâncias tóxicas produzidas pelo fumo do tabaco seriam filtradas por um recipiente de água acoplado em sua estrutura (Martins et al., 2014; WHO Study Group on Tobacco Product Regulation [WHO TobReg], 2015). Possivelmente, esse relato antigo é responsável pela crença de que o narguilé é menos prejudicial à saúde que outras formas de utilização do tabaco, no entanto, ao longo dos anos estudos comprovaram os danos advindos desta forma de consumo de tabaco (Martins et al., 2014; Ramji et al., 2015).

No narguilé, o tabaco é utilizado na forma de tablete aromatizado, colocado e submetido a alta temperatura por meio da combustão do carvão utilizado para a queima. A fumaça produzida passa por um recipiente que possui água, localizado na parte inferior do narguilé e que a resfria, to rnando, segundo os usuários, a inalação aparentemente mais agradável. Cada sessão de narguilé tem em média de 45 a 60 minutos de duração, o que representa uma exposição equivalente à fumaça de 100200 cigarros, oferecendo assim maiores níveis de nicotina e maior exposição a monóxido de carbono (Araújo et al., 2019).

Nesse contexto, a disseminação (aumento na utilização) do narguilé em várias regiões do mundo é preocupante, pois existem evidências de que seu uso, assim como o uso do tabaco do cigarro, a longo prazo pode causar danos à saúde, como câncer, doença respiratória, problemas cardíacos, infertilidade masculina, baixo peso ao nascer, comprometimento da saúde mental, dependência química e problemas periodontais (Akl et al., 2010; Luzzi et al., 2007; Morton et al., 2014; WHO, 2015).

Outra preocupação é que em uma "roda de narguilé" a quantidade de fumaça inalada por uma pessoa é muito maior do que fumar isoladamente um cigarro, pois o indivíduo age como fumante ativo e passivo ao mesmo tempo (Sorrentino et al., 2014; WHO TobReg, 2015). Além disso, os usuários compartilham o mesmo bocal, ato que facilita a propagação de doenças transmissíveis, como herpes, hepatite, COVID-19, tuberculose e, também, algumas outras doenças respiratórias (Akl et al., 2010; Instituto Nacional de Câncer [INCA], 2013; Martins et al., 2014; WHO, 2015; WHO Framework Convention on Tobacco Control [FCTC], 2020).

Em relação aos micro-organismos presentes nos componentes do narguilé, Altindis et al. (2020) realizou um estudo onde observou a presença de diversas bactérias e fungos nesses dispositivos. Em geral, constatou-se uma predominância de 
bactérias Gram-positivas nas amostras avaliadas, dentre as quais apresentaram maior prevalência: Estafilococos coagulasenegativa e Staphylococus aureus. A Escherichia coli e a Klebsiella pneumoniae foram as bactérias Gram-negativas encontradas com maior frequência durante as análises, sobretudo nos jarros dos narguilés (Altindis et al., 2020). Lopes et al. (2020) em seu estudo verificaram, ainda, a presença da Enterobacter aerogenes, a qual representa um risco grave à saúde do usuário de narguilé, uma vez que essa bactéria apresenta elevado potencial de indução a infecções respiratórias.

Atualmente, foi identificado que o consumo de tabaco aumenta o risco dos danos pulmonares causados pela infecção da COVID-19, sendo que fumantes com COVID-19 apresentam 3,25 vezes mais chances de desenvolver quadros mais graves da doença do que os indivíduos não fumantes (Guan et al., 2020).

No Brasil, poucos estudos sobre o consumo de tabaco através narguilé foram realizados. No entanto, dados fornecidos pela pesquisa nacional de saúde do escolar no Brasil em 2015, realizada com 102.301 alunos do nono ano do ensino fundamental, identificou que cerca de $24 \%$ utilizaram produtos do tabaco (cigarrilha, narguilé ou rapé) de modo usual nos 30 dias que antecederam a pesquisa, e as maiores prevalências foram encontradas na região Centro-Oeste (10\%) e região Sul (9,6\%) (Instituto Brasileiro de Geografia e Estatística [IBGE], 2016).

Na pesquisa realizada por Bertoni et al. (2019), abrangendo a população geral, revelou uma prevalência do uso de narguilé igual a 1,65\%. Esse mesmo estudo apontou que o narguilé é mais utilizado nas macrorregiões Sul/Sudeste $(2,46 \%) \mathrm{em}$ relação às regiões Centro-Oeste $(2,21 \%)$ e Norte/Nordeste $(0,22 \%)$. Além disso, essa prática se mostrou mais prevalente na população do sexo masculino $(2,36 \%)$ em relação à do sexo feminino $(0,97 \%)$ e menos prevalente na população heterossexual $(1,55 \%)$ em relação à não-heterossexual $(4,20 \%)$. Quanto à prevalência do uso do narguilé em diferentes classes socioeconômicas, essa pesquisa revelou, ainda, que essa prática é mais comum entre pessoas com renda familiar mensal superior a $\mathrm{R} \$ 6.000,00$ (seis mil reais).

Com o intuito de auxiliar a saúde pública e visando conter a proliferação do uso do narguilé, algumas leis já foram criadas. Em 21 de Outubro de 2009, a Lei n. 13.799 (2009) teve como objetivo proibir a venda de narguilé para menores de 18 anos no estado de São Paulo (Martins et al., 2014). Nas cidades de Medianeira e Cascavel, ambas no Oeste do Paraná, a Lei n. 300 (2013) e a Lei n. 6233 (2013) respectivamente, penalizam quem estiver fumando em lugares públicos. Segundo a Câmara Municipal de Cascavel (2014), há relatos de que na região do lago municipal o uso do narguilé diminuiu em 90\% após a implantação da lei.

Neste contexto, um dos objetivos deste estudo foi aplicar um questionário da primeira à última série do Curso de Odontologia da Universidade Estadual do Oeste do Paraná (UNIOESTE), para analisar a prevalência de experimentação e do uso de narguilé entre os acadêmicos, bem como o conhecimento a respeito do consumo. Além disso, a pesquisa objetivou, também, verificar qual a visão que estes futuros cirurgiões dentistas possuem frente ao papel que devem exercer como profissionais de saúde nas ações antitabagistas.

\section{Metodologia}

Foi realizado um estudo transversal e quantitativo por meio da aplicação de um questionário autoadministrado entre os acadêmicos do $1^{\circ}$ ao $5^{\circ}$ ano do curso de Odontologia UNIOESTE no ano de 2016. De acordo com Estrela (2005), os estudos transversais são frequentemente chamados de estudos de prevalência e objetivam avaliar o estado de um indivíduo com relação à presença ou ausência da exposição a algum fator de risco em um determinado momento, conforme foi realizado em nosso estudo. O projeto de pesquisa foi aprovado pelo Comitê de Ética e Pesquisa da UNIOESTE, com o CAAE: 56029416.6.0000.01.07. 
O questionário autoaplicável utilizado foi composto por 16 perguntas que buscaram identificar: o hábito dos participantes com relação ao uso do narguilé e influência para o uso do mesmo, o seu conhecimento sobre os riscos causados pelo narguilé à saúde e a opinião sobre a atitude dos profissionais de saúde frente ao consumo de tabaco (Quadro 1).

Quadro 1. Questionário sobre hábitos de fumar, conhecimentos e opiniões acerca do narguilé.

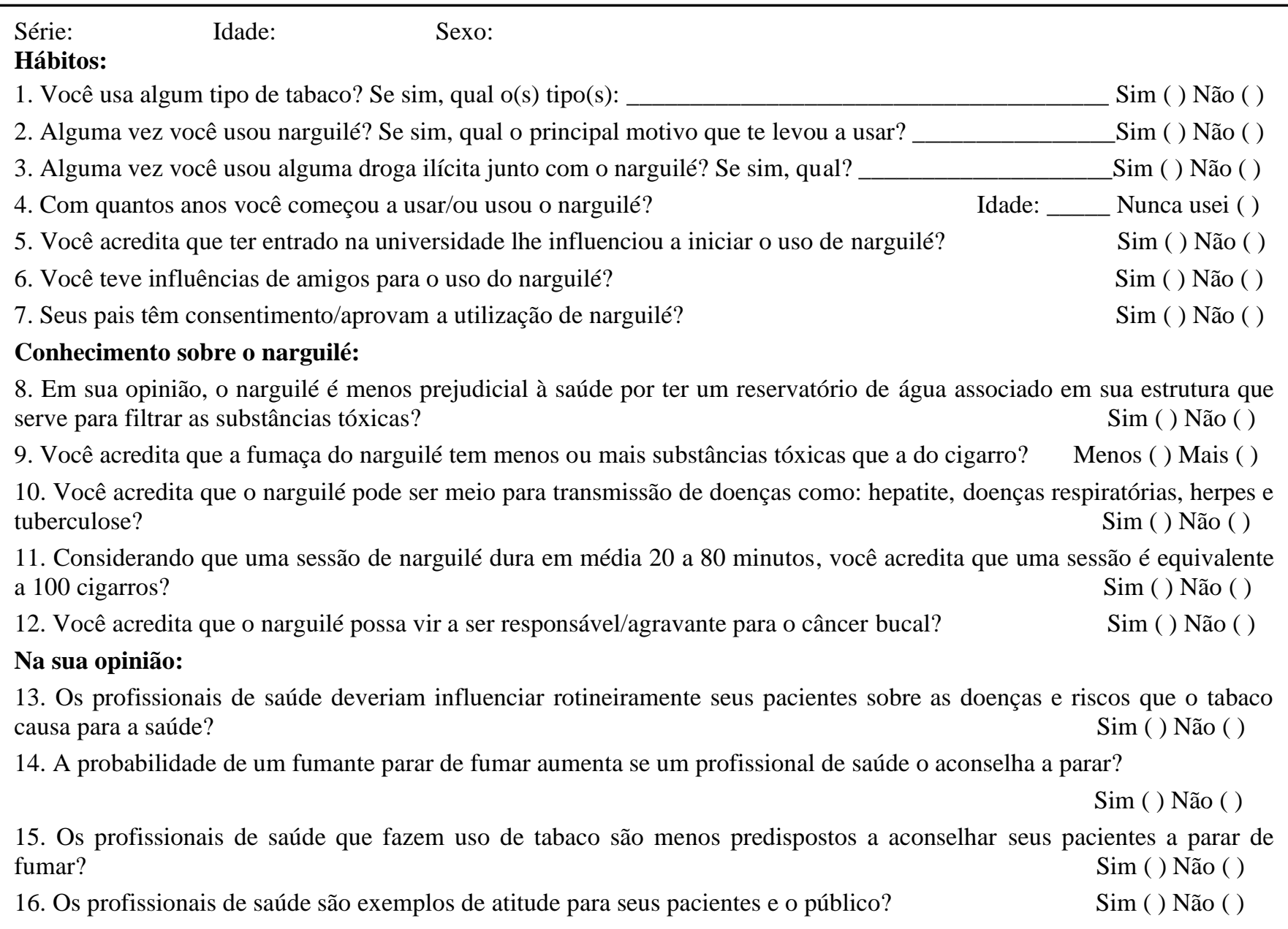

Fonte: Autores.

A participação na pesquisa foi voluntária e os participantes assinaram o TCLE (Termo de Consentimento Livre e Esclarecido). Para aplicação do questionário, foi solicitado ao coordenador do curso de Odontologia autorização para utilizar 15 minutos de uma aula da graduação a fim de explicar aos alunos sobre a importância do estudo.

Os dados coletados foram organizados em planilhas eletrônicas (Excel 2007, Microsoft, EUA) sendo avaliados com o teste Qui-Quadrado de Pearson ou Exato de Fischer, através do software IMB SPSS Statistics, versão 20.0.

\section{Resultados}

Dos 196 acadêmicos matriculados em todas as séries do curso de Odontologia da UNIOESTE no ano de 2016, participaram deste estudo 160 alunos (82\%). A média de idade dos participantes foi de 21,34 anos, variando de 17 a 38 anos, sendo que 73\% (117/160) se encontravam na faixa etária dos 20 aos 24 anos. Os participantes do sexo feminino representaram $75 \%$ (120/160) da amostra. Dentre os entrevistados, 32\% (51/160) fazem o uso contínuo de algum tipo de tabaco, sendo o narguilé utilizado por 96\% (49/51) como pode ser observado Figura 1. 
Figura 1. Distribuição do tipo de tabaco utilizado pelos participantes da pesquisa ( $\mathrm{n}=51)$.

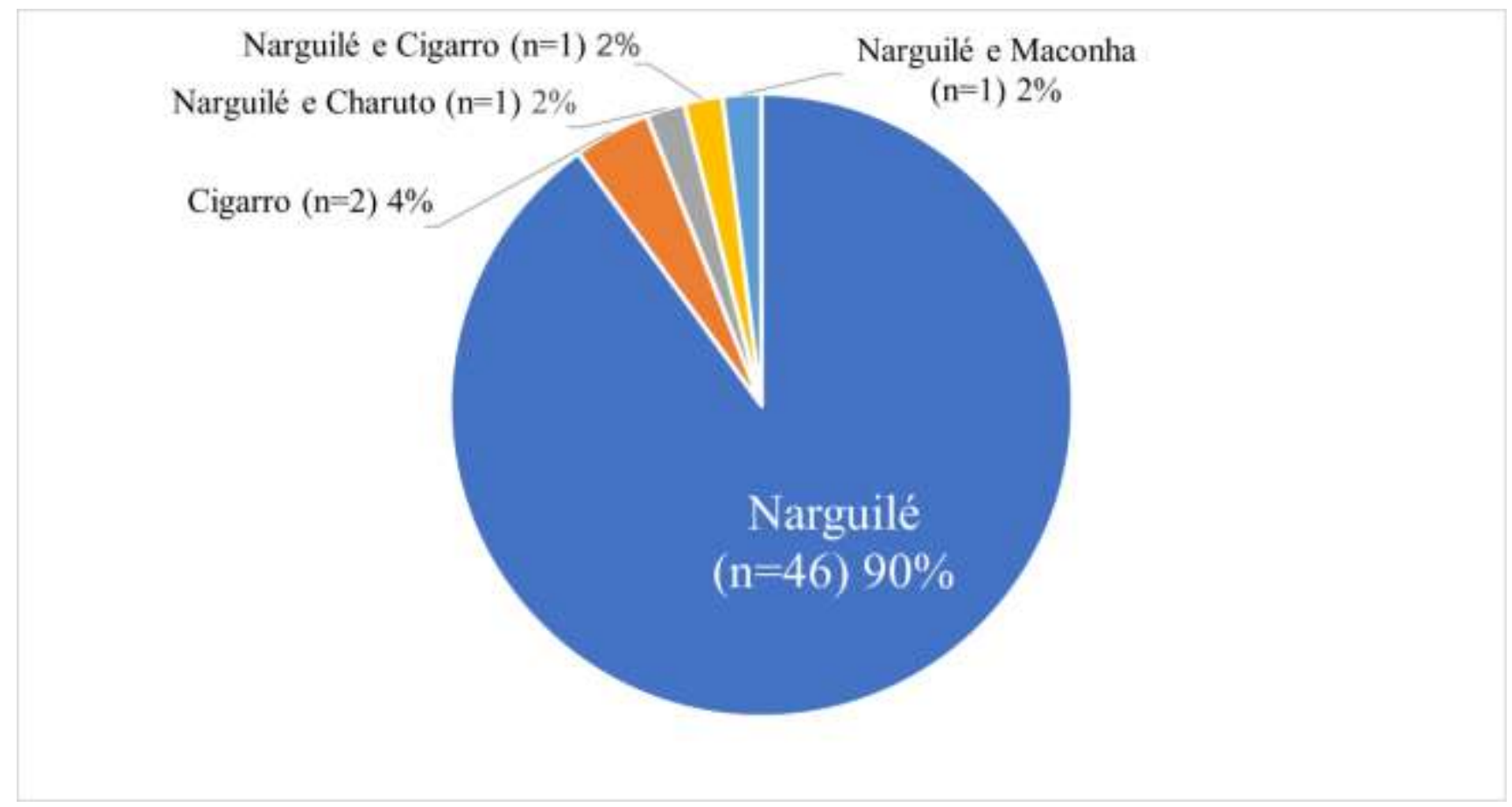

Fonte: Autores.

$\mathrm{Na}$ amostra entrevistada, 69\% (111/160) dos acadêmicos já experimentaram o narguilé, sendo que 45\% (50/111) foram motivados pela curiosidade em conhecer essa forma de utilização de tabaco (Figura 2).

Neste estudo, 59\% (66/111) dos acadêmicos que já fumaram narguilé iniciaram o uso na faixa etária entre 17 e 19 anos e $41 \%$ (45/111) entre 20 e 24 anos. Dentre esses, 23\% (25/111) informaram que entrar para a universidade foi um fator que influenciou o uso do narguilé e 77\% (85/111) responderam que um dos motivos que os levou a experimentar o narguilé foi a influência dos amigos. A ausência do consentimento dos pais acerca do uso do narguilé foi observada em 71\% (79/111) da amostra que já fez uso da referida substância e 8\% (9/111) afirmaram que já utilizaram drogas ilícitas junto com o narguilé.

Sobre o conhecimento acerca do narguilé, 52\% (83/160) afirmaram que a fumaça possuiu menos substâncias químicas que a do cigarro, 59\% (94/160) não acreditam que utilizar o narguilé seja equivalente a consumir 100 cigarros ao mesmo tempo e 86\% (137/160) confirmam que o reservatório de água acoplado em sua estrutura não diminui os danos à saúde. 
Figura 2. Principal motivo que levou os participantes a experimentarem narguilé ( $\mathrm{n}=111)$.

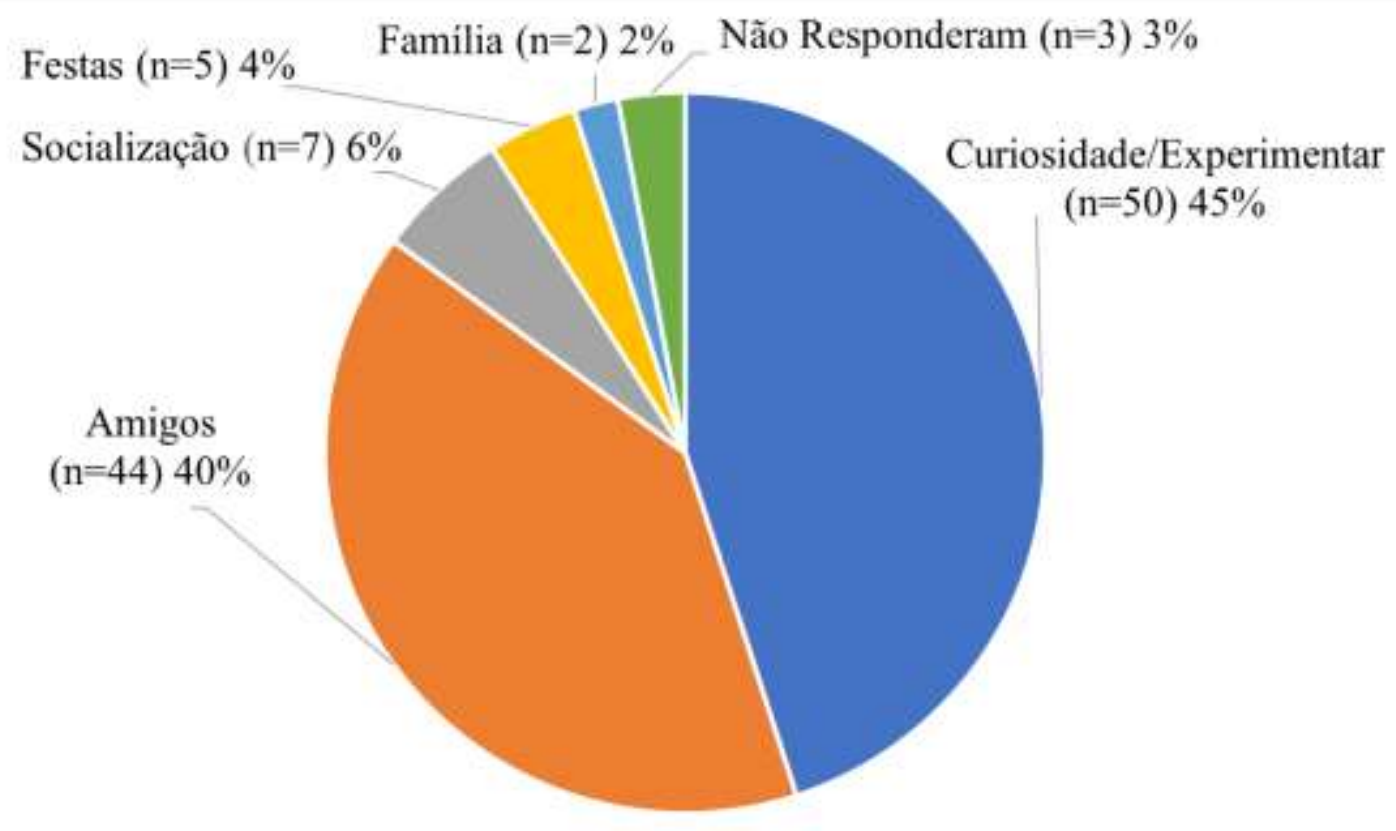

Fonte: Autores.

Entre os referidos acadêmicos, 91\% (145/160) afirmam que esta forma de utilizar tabaco pode ser meio de transmissão de doenças, dentre elas: hepatite, doenças respiratórias, herpes e tuberculose. Além disso, nesta amostra a associação do narguilé com o câncer bucal foi referida por 89\% (143/160) dos entrevistados.

No que se refere às questões relacionadas à influência do profissional da saúde no controle do uso do tabaco, $99 \%$ (159/160) da amostra considerou que esses profissionais devem orientar rotineiramente seus pacientes sobre as doenças e riscos do tabaco para a saúde e $89 \%$ (142/160) acreditam que o aconselhamento pelos profissionais de saúde aumenta a probabilidade de os fumantes cessarem o hábito. Além disso, 56\% (90/160) dos entrevistados consideram que o fato do profissional da saúde fazer o uso do tabaco o torna menos predisposto a aconselhar os seus pacientes e 86\% (138/160) acreditam que os profissionais da saúde são exemplos de atitude para seus pacientes e público. Nesse sentido, o presente estudo observou a correlação positiva $(p=0,00)$ entre a influência dos amigos e o uso do narguilé.

\section{Discussão}

Há muitos anos que o tabagismo é considerado um grande problema para a saúde pública, devido a sua agressividade para o organismo humano e pela elevada dependência que causa aos usuários que, apesar de ser evitável, é considerada a segunda maior causa de morte no mundo (Luzzi et al., 2007; WHO 2015). Atualmente, estudos apontam que o consumo de cigarro vem diminuindo, sendo substituído por outras formas de usar o tabaco, entre elas, o narguilé (Beckert et al., 2016; Farias et al., 2015; INCA, 2013; Jawad et al., 2013; Martins et al., 2014; Rossi, 2013; Szklo et al., 2011; WHO, 2015).

Segundo dados do Observatório da Política Nacional de Controle do Tabaco (2019), a prevalência de fumantes de cigarro no Brasil teve uma queda significativa ao longo dos anos, passando de 15,6\% em 2006 para 9\% em 2018 (INCA, 2019). Estima-se que cerca de 212 mil brasileiros admitem usar narguilé (Kopko, 2015). No presente estudo, observou-se que 69\% (111/160) dos estudantes de odontologia entrevistados já experimentaram narguilé, resultado semelhante ao encontrado em pesquisa com estudantes de medicina no Líbano (65\%), país em que o uso do narguilé faz parte da cultura local (Jawad et al., 2013). 
No Brasil, os dados do Ministério da Saúde mostram que o uso do narguilé é maior na região Sul, fato confirmado por nosso estudo, visto que uma pesquisa realizada na Universidade de São Paulo identificou 47\% de experimentação entre os alunos do curso de medicina (Kopko, 2015; Martins et al., 2014). Este índice é semelhante aos de escolas médicas em outros países, como na Inglaterra (52\%), Canadá (40\%) e Estados Unidos (48,4\%) (Jawad et al., 2013; Morton et al., 2014; Vanderhoek et al., 2013).

Além disso, o Global Youth Survery (1999-2008), responsável pela vigilância mundial do tabagismo em escolas, realizou uma pesquisa envolvendo mais de 500.000 estudantes do mundo inteiro, entre 13 e 15 anos, e concluiu que o uso do narguilé se mostra mais frequente, enquanto o uso do cigarro está decrescendo ou mantendo-se estável (Menezes et al., 2015). Em um estudo realizado entre acadêmicos de fisioterapia da UNIOESTE, observou-se uma prevalência de $40 \%$ do uso de narguilé por esses estudantes, enquanto apenas 1,6\% da amostra informou fazer uso do cigarro (Fachin, 2018), corroborando com as informações supracitadas. O declínio de usuários de cigarro pode estar relacionado com as políticas públicas voltadas para a conscientização dos efeitos nocivos do mesmo e pela implantação das leis antifumo em alguns países, como no Brasil (Issa \& Lopes, 2014; Martins et al., 2013; Rossi, 2013).

O presente estudo confirma esta realidade, pois dos $32 \%$ (51/160) dos acadêmicos que fazem uso do tabaco rotineiramente, $96 \%$ (49/51) usam o narguilé, enquanto apenas 5,9\% (3/51) fazem o uso do cigarro. Já entre os estudantes de odontologia de uma universidade de Curitiba, o uso do narguilé foi observado em 66,23\% dos acadêmicos que usam tabaco rotineiramente (Beckert et al., 2013). Esta realidade não está apenas limitada ao nosso país, no Canadá, por exemplo, os alunos de medicina utilizam o dobro de narguilé quando comparado com cigarro (Vanderhoek et al., 2013).

A ascensão do uso do narguilé pode estar vinculada com a interação e socialização entre as pessoas, pelo odor agradável que o instrumento proporciona ao ambiente, sua fumaça ter gosto prazeroso ao paladar, uso de essência aromatizada e por ser encontrado em bares, casas noturnas e cafés (Auf et al., 2012; Beckert et al., 2016; INCA, 2013; Martins et al., 2014; Menezes et al., 2015; Morton et al., 2014; Szklo et al., 2011; WHO TobReg, 2015). Observou-se nesse estudo que dos 69\% (111/160) dos acadêmicos que já experimentaram o narguilé, 77\% (85/111) deles foram influenciados pelos amigos. Esta pesquisa corrobora com o estudo de Beckert et al. (2016), em que 71,43\% dos estudantes de odontologia relataram ter sido influenciados por amigos para o uso do narguilé, e com a pesquisa de Fachin (2018) com estudantes de fisioterapia, que revelou que $89 \%$ dos acadêmicos de fisioterapia fizeram uso do narguilé influenciados por amigos. Para Aljarrah et al. (2009), o ambiente social com amigos é um dos fatores atribuídos a sua propagação dramática.

Issa e Lopes (2014) ressaltam em seu estudo que os jovens possuem ausência de maturidade para inibir seus atos de impulsividade, e que nesta fase são influenciados pelo fascínio de experimentar, com isso a indústria do tabaco tem ao seu lado fatores que facilitam a propagação de seus produtos. Para Rossi (2013), os jovens são muito vulneráveis e o ato de fumar se dá justamente em decorrência da curiosidade. Em nossa pesquisa isso se confirma, uma vez que 59\% (66/111) dos entrevistados que experimentaram o narguilé iniciaram o uso na adolescência, entre 14 e 19 anos, e 41\% (45/111) entre 20 e 24 anos e a curiosidade foi relatada por 45\% (50/111) dos entrevistados. No estudo de Fachin (2018), 70\% referiram usar narguilé antes dos 18 anos.

Em nosso estudo, 52\% (83/160) acreditam que a fumaça do cigarro possui mais substâncias tóxicas quando comparada com o narguilé. Esse resultado é semelhante aos encontrados por Paiva et al. (2020), em que 61,5\% dos usuários acreditam que o narguilé seja menos prejudicial que o cigarro, e por Fachin (2018), no qual 57\% dos acadêmicos disseram acreditar que o narguilé possui menos substâncias tóxicas que o cigarro. Embora muitas pessoas acreditem que usar narguilé seja uma forma mais segura para o uso do tabaco, estudos comprovam que o percentual de monóxido de carbono presente na fumaça do narguilé é muito maior do que no cigarro (Aljarrah et al., 2009; Martins et al., 2014; Sorrentino et al., 2014; Viegas, 2008; WHO TobReg, 2015). É importante ressaltar, ainda, que na análise da fumaça do narguilé são encontradas substâncias 
tóxicas dentre as quais estão: nicotina, alcatrão, metais pesados, arsênio, benzopireno, níquel, cobalto, berílio, cromo e chumbo em quantidades superiores à fumaça do cigarro (Consolaro et al., 2010; Viegas, 2008; WHO TobReg, 2015). Além disso, a concentração de nicotina presente no sangue após a sessão de narguilé é maior comparada com um único cigarro e suficiente para causar o vício (Morton et al., 2014). Em uma pesquisa em Aleppo, na Síria, sobre dependência, dos 268 usuários, 28\% queriam largar o vício e 59\% tinham tido tentativas malsucedidas de abandonar o uso do narguilé (WHO TobReg, 2015). Outro estudo realizado no Egito revelou que os usuários de narguilé preenchem critérios semelhantes aos usuários de cigarro quanto à dependência da nicotina (Martins et al., 2014).

Em uma análise comparativa da quantidade de inalação da fumaça do cigarro e do narguilé, é possível destacar que, no primeiro, os fumantes inalam entre 8 e 12 baforadas de fumaça com 40-75 mL cada, inalando 0,5-0,6 $\mathrm{L}$ de fumaça por cigarro em aproximadamente 6 minutos. Em contrapartida, uma sessão de narguilé dura em média de 20 a 80 minutos, com isso, o fumante inala 50-200 baforadas num total de 0,5-1,0 L de fumaça, podendo ser considerado que uma sessão de narguilé seja equivalente a 100 cigarros ou mais (Aljarrah et al., 2009; Viegas, 2008). Apesar dos estudos disponíveis, identificamos em nossa pesquisa que 59\% (94/160) dos entrevistados discordam deste fato.

A temperatura da queima do tabaco no cigarro é cerca de $450^{\circ}$, enquanto no narguilé essa temperatura é igual a $900^{\circ}$, o que pode produzir diferentes tipos de substâncias químicas que são nocivas (Aljarrah et al., 2009). Ademais, os produtos químicos presentes na fumaça do narguilé são classificados como cancerígenos pela Agência Internacional de Investigação do Câncer (WHO TobReg, 2015). Estas substâncias são perigosas e têm sido associadas a algumas doenças, como por exemplo, na Índia, em que evidências, a partir de junho de 2014, associaram-nas ao câncer oral, na República Islâmica do Irã três estudos vincularam-nas ao câncer de esôfago e outras seis pesquisas realizadas na China, Índia e Tunísia relacionaram-nas com o câncer do pulmão (WHO TobReg, 2015). Apesar destes dados, neste estudo 11\% (17/160) dos entrevistados ainda acreditam que o narguilé não pode ser agravante ou precursor ao câncer bucal. Além disso, Akl et al. (2010) em revisão sistemática de 24 estudos, concluíram que o tabagismo por meio do narguilé tem resultados significantes quando relacionados ao câncer de pulmão, baixo peso ao nascer, doença respiratória e doença periodontal.

Além das doenças que podem ser adquiridas a longo prazo, sabe-se que, pelo fato de os usuários compartilharem o mesmo bocal, o narguilé pode ser meio de transmissão de doenças, como herpes, hepatite, tuberculose e outras doenças respiratórias (Aljarrh et al., 2009; Farias et al., 2015; INCA, 2013; Martins et al., 2014; Menezes et al., 2015). Nesta amostra, 92\% (102/111) dos acadêmicos que experimentaram narguilé têm consciência disso, porém ter o conhecimento não foi o suficiente para que não experimentassem, colocando-se, então, em risco. Cabe salientar, mesmo não sendo objeto de estudo desta pesquisa, que a intoxicação momentânea por monóxido de carbono pode causar dores de cabeça, tonturas, náuseas, palpitações e até mesmo síncope (Akl et al., 2010; Aljarrah et al., 2009; Martins et al., 2014; WHO TobReg, 2015).

Quando questionados sobre a influência dos profissionais da saúde em políticas antitabagistas, 86\% (138/160) dos acadêmicos acreditam ser importantes para a sociedade e 56\% (90/160) relatam que profissionais fumantes são menos predispostos a aconselhar seus pacientes a pararem de fumar. Em uma pesquisa realizada com os estudantes de fisioterapia da mesma instituição, 59\% dos entrevistados responderam que profissionais fumantes são menos predispostos a orientar seus pacientes a cessar o vício (Fachin, 2018). Dos entrevistados em nosso estudo, 99\% (159/160) responderam que os profissionais da saúde devem orientar rotineiramente seus pacientes sobre as doenças e riscos do tabaco para a saúde tendo, esse resultado, semelhança com os resultados da pesquisa de Martins et al. (2014), em que todos os futuros médicos acreditavam que os profissionais da saúde devem aconselhar seus pacientes para parar de fumar.

A atitude dos pais é de suma importância para o controle do uso do tabaco, embora para algumas culturas, como na comunidade árabe, fumar narguilé é uma atividade social aceitável entre adolescentes e dentro das reuniões familiares (Roohafza et al., 2015). Dos acadêmicos que relataram já ter experimentado o narguilé, 71\% (79/111) declararam que seus pais 
são contra a utilização, ou seja, mesmo sem a permissão dos responsáveis não houve controle do uso. Não foi o objeto de estudo saber a reação dos pais frente ao hábito, mas é interessante salientar que Roohafza et al. (2015) expõe que punições, chamadas de atenção e conversas a respeito do tabaco podem reduzir o consumo de 60 a $71 \%$.

Neste estudo, percebe-se que a grande parte dos acadêmicos tem consciência de que o uso de narguilé é prejudicial à saúde, porém isto não foi o suficiente para impedir que os mesmos o experimentassem. Dos 69\% (111/160) dos entrevistados que experimentaram narguilé, quase metade (44\%) faz o seu uso atualmente. Isto é preocupante e merece atenção especial da universidade, pois a tendência do usuário é aumentar o hábito a cada dia, tornando-se um dependente de nicotina e abrindo a possibilidade para utilização de outras drogas (Farias et al., 2015; Issa \& Lopes, 2014; Rossi, 2013; WHO TobReg, 2015), como foi identificado em $8 \%(9 / 111)$ dos jovens neste estudo.

\section{Conclusão}

Neste estudo, 69\% (111/160) dos acadêmicos de odontologia experimentaram narguilé, sendo que desses, $41 \%$ (46/111) fazem o uso rotineiramente, enquanto os demais tiveram apenas um único contato.

No teste de correlação, a experimentação do narguilé apresentou correlação positiva com a influência dos amigos $(\mathrm{p}=0,00)$, e tanto os acadêmicos que experimentaram narguilé e os que não, acreditam que os profissionais de saúde são exemplos de atitude para seus pacientes.

Os resultados deste estudo indicam a necessidade de ser realizada uma discussão ampla com a comunidade acadêmica, com o objetivo de esclarecer aos jovens sobre os malefícios do narguilé e suas consequências para a saúde, e contribuir para que os mesmos desenvolvam o senso crítico que possibilite que as influências da sociedade a qual eles fazem parte não interfiram nas suas escolhas.

Sugere-se, para trabalhos futuros, a aplicação de questionários com enfoque em outras formas de utilização do tabaco, como por exemplo o cigarro eletrônico.

\section{Referências}

Akl, E. A., Gaddam, S., Gunukula, S. K., Honeine, R., Jaoude, P. A., \& Irani, J. (2010). The effects of waterpipe tobacco smoking on health outcomes: a systematic review. International journal of epidemiology, 39(3), 834-857. https://doi.org/10.1093/ije/dyq002

Aljarrah, K., Ababneh, Z. Q., \& Al-Delaimy, W. K. (2009). Perceptions of hookah smoking harmfulness: predictors and characteristics among current hookah users. Tobacco induced diseases, 5(1), 1-7. https://doi.org/10.1186/1617-9625-5-16

Altindis, M., Koroglu, M., Demiray, T., Yilmaz, K., Baran Inci, M., Olmez, M., Altindis, S., \& Erkorkmaz, U. (2020). Microbial contamination and infection risks of narghile besides hazards of tobacco. Central European journal of public health, 28(1), 74-78. https://doi.org/10.21101/cejph.a5814

Araújo, R. D. S., Milhomem, Y. D. O., Pereira, H. F. S., \& Silva Junior, J. L. R. D. (2019). Fatores relacionados ao consumo do narguilé entre estudantes de medicina. Jornal Brasileiro de Pneumologia, 45(5), e20180184. https://doi.org/10.1590/1806-3713/e20180184

Auf, R. A., Radwan, G. N., Loffredo, C. A., El Setouhy, M., Israel, E., \& Mohamed, M. K. (2012). Assessment of tobacco dependence in waterpipe smokers in Egypt. The international journal of tuberculosis and lung disease, 16(1), 132-137. https://doi.org/10.5588/ijtld.11.0457

Beckert, N., Moysés, S., Cruz, R., Gutoski, L., \& Scarinci, I. (2016). Características do uso de produtos derivados do tabaco entre universitários do curso de Odontologia em uma Universidade de Curitiba. Revista de Odontologia da UNESP, 45(1), 7-14. https://doi.org/10.1590/1807-2577.10015

Bertoni, N., Szklo, A., De Boni, R., Coutinho, C., Vasconcellos, M., Silva, P. N., De Almeida, L. M., \& Bastos, F. I. (2019). Electronic cigarettes and narghile users in Brazil: Do they differ from cigarettes smokers? Addictive behaviors, 98, 106007. https://doi.org/10.1016/j.addbeh.2019.05.031

Câmara Municipal de Cascavel. (2014). Em vigor: lei que proíbe o uso de narguilé em locais públicos completa um ano. https://www.camaracascavel.pr.gov.br/noticias-xml/item/4690-em-vigor-lei-que-proibe-narguile-em-locais-publicos-completa-um-ano.html

Consolaro, R. B., Demathé, A., Biasoli, É. R., \& Miyahara, G. I. (2010). O tabaco é um dos principais fatores etiológicos do câncer bucal: conceitos atuais. Revista Odontológica de Araçatuba, 31(2), 63-67.

Estrela, C. (2005). Metodologia científica: ciência, ensino, pesquisa (2a ed.). São Paulo: Artes Médicas.

Fachin, F. G. (2018). Estudo e prevalência de fumar narguilé entre estudantes de fisioterapia da Universidade Estadual do Oeste do Paraná. Trabalho de conclusão de curso, Universidade Estadual do Oeste do Paraná, Cascavel, Paraná, Brasil. 
Farias, L. F., Sorato, A., \& Arruda, V. (2015). Cigarro e Narguilê: o que os acadêmicos pensam sobre essas drogas?. Enciclopédia Biosfera, 11(22), 33673380. http://dx.doi.org/10.18677/Enciclopedia_Biosfera_2015_050

Guan, W. J., Ni, Z. Y., Hu, Y., Liang, W. H., Ou, C. Q., He, J. X., Liu, L., Shan, H., Lei, C. L., Hui, D. S. C., Du, B., Li, L. J., Zeng, G., Yuen, K. Y., Chen, R. C., Tang, C. L., Wang, T., Chen, P. V., Xiang, J., Li, S. Y., Wang, J. L., Liang, Z. J., Peng, Y. X., Wei, L., Liu, Y., Hu, Y. H., Peng, P., Wang, J. M., Liu, J. Y., Chen, Z., Li, G., Zheng, Z. J., Qiu, S. Q., Luo, J., Ye, C. J., Zhu, S. Y., \& Zhong, N. S. (2020). Clinical characteristics of coronavirus disease 2019 in China. New England journal of medicine, 382(18), 1708-1720. https://doi.org/10.1056/NEJMoa2002032

Instituto Brasileiro de Geografia e Estatística. (2016). Pesquisa nacional de saúde do escolar: 2015. Rio de Janeiro: IBGE.

Instituto Nacional de Câncer. (2013). O que é narguilé?. http://www.inca.gov.br/wcm/dncf/2013/o-que-e-narguile.asp

Instituto Nacional de Câncer. (2019). Informativo da Comissão Nacional para implementação da Convenção-Quadro da OMS para o Controle do Tabaco no Brasil, $n^{\circ}$ 05, ano 2019. https://www.inca.gov.br/sites/ufu.sti.inca.local/files//media/document//informativo-conicq-agosto-setembro-outubro-2019.pdf

Issa, J. S., \& Lopes, G. M. (2014). Muito além do tabaco. Jornal Brasileiro de Pneumologia, 40(2), 99-101.

Jawad, M., Abass, J., Hariri, A., Rajasooriar, K. G., Salmasi, H., Millett, C., \& Hamilton, F. L. (2013). Waterpipe smoking: prevalence and attitudes among medical students in London. The International journal of tuberculosis and lung disease, 17(1), 137-140. https://doi.org/10.5588/ijtld.12.0175

Kopko, G. (2015). Mais de 212 mil brasileiros admitem usar narguilé. https://www.unasus.gov.br/noticia/mais-de-212-mil-brasileiros-admitem-usarnarguile

Lei n. 13.799, de 21 de outubro de 2009 (2009). Proíbe a venda de narguilé aos menores de 18 anos.São Paulo, SP. https://www.al.sp.gov.br/repositorio/legislacao/lei/2009/lei-13779-21.10.2009.html

Lei n. 6.233, de 1 de julho de 2013 (2013). Fica proíbido o uso e a venda de cachimbo conhecido como "narguile" aos menores de 18 anos, e dá outras providências. Cascavel, PR. Recuperado em 18 maio, 2021, de http://leismunicipa.is/ufkih

Lei n. 300, de 21 de novembro de 2013 (2013). Dispõe sobre a comercialização e o uso do Narguilé em local público, no âmbito do Município de Medianeira, Estado do Paraná, e dá outras providências. Medianeira,://www.camaramedianeira.pr.gov.br/downloads/lo2013_300.pdf

Lopes, A. R., Hoscheid, J., \& Miranda, N. (2020). Análise microbiológica dos componentes do narguilé. Research, Society and Development, 9(10), e1769108431. https://doi.org/10.33448/rsd-v9i10.8431

Luzzi, L. I. T., Greghi, S. L. A., Passanezi, E., Sant'Ana, A. C. P., Lauris, J. R. P., \& Cestari, T. M. (2007). Evaluation of clinical periodontal conditions in smokers and non-smokers. Journal of Applied Oral Science, 15(6), 512-517. https://doi.org/10.1590/S1678-77572007000600011

Martins, S. R., Paceli, R. B., Bussacos, M. A., Fernandes, F. L. A., Prado, G. F., Lombardi, E. M. S., Terra-Filho, M., \& Santos, U. P. (2014). Experimentação de e conhecimento sobre narguilé entre estudantes de medicina de uma importante universidade do Brasil. Jornal Brasileiro de Pneumologia, 40(2), 102-110. https://doi.org/10.1590/S1806-37132014000200002

Menezes, A. M. B., Wehrmeister, F. C., Horta, B. L., Szwarcwald, C. L., Vieira, M. L., \& Malta, D. C. (2015). Frequência do uso de narguilé em adultos e sua distribuição conforme características sociodemográficas, moradia urbana ou rural e unidades federativas: Pesquisa Nacional de Saúde (PNS), 2013. Revista Brasileira de Epidemiologia, 18, 57-67. https://doi.org/10.1590/1980-5497201500060006

Morton, J., Song, Y., Fouad, H., Awa, F. E., Abou El Naga, R., Zhao, L., Palipudi, K., \& Asma, S. (2014). Cross-country comparison of waterpipe use: nationally representative data from 13 low and middle-income countries from the Global Adult Tobacco Survey (GATS). Tobacco control, 23(5), 419-427. http://dx.doi.org/10.1136/tobaccocontrol-2012-050841

Paiva, M. O., de Lima, A. B., Vaz, R. S., \& Granemann, P. (2020). Prevalência do uso de narguilé entre universitários da área da saúde. Revista de Medicina, 99(4), 335-341. https://doi.org/10.11606/issn.1679-9836.v99i4p335-341

Ramji, R., Arnetz, J., Nilsson, M., Jamil, H., Norström, F., Maziak, W., Ywonne W.,\& Arnetz, B. (2015). Determinants of waterpipe use amongst adolescents in Northern Sweden: a survey of use pattern, risk perception, and environmental factors. BMC research notes, 8(1), 441. https://doi.org/10.1186/s13104-015$1413-4$

Roohafza, H., Kasaei, Z., Heidari, K., Omidi, R., Alinia, T., Naji, M., Jaberifar, M., \& Sadeghi, M. (2015). Better view on attitudes and perceived parental reactions behind waterpipe smoking among Iranian students. Journal of research in medical sciences: the official journal of Isfahan University of Medical Sciences, 20(11), 1032-1038. https://doi.org/10.4103/1735-1995.172812

Rossi, L. C. D. C. (2013). Movendo-se entre a experimentação e a dependência de drogas: o autocontrole do adolescente como componente interveniente. Tese de doutorado, Universidade Estadual Paulista 'Júlio de Mesquita Filho”, Botucatu, SP, Brasil. https://repositorio.unesp.br/handle/11449/106060

Sorrentino, M. A. S., Alvarez, M. C., Santos, R. S., Guerra, S. E. P., \& Gugliano, E. B. (2014). O uso do narguilé e o efeito nocivo químico físico e biológico causado pela liberação das toxinas do tabaco: avaliação do uso do cigarro do narguilé em umainstituição de ensino superior na cidade de Santos/SP. http://aretesaudehumana.blogspot.com.br/2013/05/o-uso-do-narguile-e-o-efeito-nocivo.html

Szklo, A. S., Sampaio, M. M. A., Fernandes, E. M., \& Almeida, L. M. D. (2011). Perfil de consumo de outros produtos de tabaco fumado entre estudantes de três cidades brasileiras: há motivo de preocupação?. Cadernos de Saúde Pública, 27(11), 2271-2275.

Vanderhoek, A. J., Hammal, F., Chappell, A., Wild, T. C., Raupach, T., \& Finegan, B. A. (2013). Future physicians and tobacco: an online survey of the habits, beliefs and knowledge base of medical students at a Canadian University. Tobacco induced diseases, 11(1), 9. https://doi.org/10.1186/1617-9625-11-9

Viegas, C. A. D. A. (2008). Formas não habituais de uso do tabaco. Jornal Brasileiro de Pneumologia, 34(12), 1069-1073. http://dx.doi.org/10.1590/S180637132008001200013 
Research, Society and Development, v. 11, n. 1, e30811124805, 2022

(CC BY 4.0) | ISSN 2525-3409 | DOI: http://dx.doi.org/10.33448/rsd-v11i1.24805

WHO Framework Convention on Tobacco Control. (2020). Increased risk of COVID-19 infection amongst smokers and amongst waterpipe users. de https://untobaccocontrol.org/kh/waterpipes/covid-19/

WHO Study Group on Tobacco Product Regulation. (2015). Advisory note: Waterpipe tobacco smoking: health effects, research needs and recommended actions for regulators (2a ed.). Geneva: World Health Organization. 